\title{
Cis-dicarbonyl binding at cobalt and iron porphyrins with saddle-shape conformation
}

\author{
Knud Seufert ${ }^{1}$, Marie-Laure Bocquet ${ }^{2}$, Willi Auwärter ${ }^{1}$, Alexander \\ Weber-Bargioni ${ }^{3}$, Joachim Reichert ${ }^{1,3}$, Nicolás Lorente $^{4} \&$ Johannes V. \\ Barth $^{1,3}$ \\ ${ }^{1}$ Physik Department E20, TU München, James-Franck Str, D-85748 \\ Garching, Germany; ${ }^{2}$ Laboratoire de Chimie, LR6, ENS Lyon, 46 allée \\ d'Italie, 69364 Lyon cedex 07, France; ${ }^{3}$ Department of Physics \& \\ Astronomy, University of British Columbia, Vancouver, BC V6T1Z4, \\ Canada; ${ }^{4}$ Centre d' Investigació en Nanociència i Nanotecnologia \\ (CSIC-ICN), Campus de la UAB, Bellaterra E-08193, Spain
}

\begin{abstract}
Diatomic molecules attached to complexed $\mathrm{Fe}$ or Co centers are significant for many biological processes. In natural systems notably metallotetrapyrrole units carry respiratory gases or provide sensing and catalytic functions. Conceiving synthetic model systems strongly helped to rationalize the pertaining chemical foundations, whereby recent work highlights the importance of the prosthetic groups' conformational flexibility as an intricate variable affecting their functional properties. Here we present simple model systems to investigate at the single-molecule level the interaction of carbon monoxide with saddle-shaped $\mathrm{Fe}$ - and Co-porphyrin conformers, which have been stabilized as two-dimensional arrays on well-defined surfaces. Using scanning tunneling microscopy we identified a novel bonding scheme expressed in tilted monocarbonyl and cis-dicarbonyl configurations at the functional metalmacrocycle unit. Density functional theory modeling reveals that the weakly bonded diatomic carbonyl adduct can effectively bridge specific pyrrole groups with the metal atom because of the pronounced saddle-shape conformation of the porphyrin cage.
\end{abstract}


The interaction of diatomic molecules with complexed transition metal centers is decisive in many biological processes. Nature notably employs metalloporphyrins with $\mathrm{Fe}$ or $\mathrm{Co}$ centers as prosthetic groups to handle respiratory gases, sensing and catalytic functions, whereby a plethora of model systems has been investigated to disentangle the underlying elementary chemical binding mechanisms. ${ }^{1,2,3}$ In recent years it became clear that the conformational flexibility of the porphyrin macrocycle sensitively interferes as a decisive factor in the regulation of the pertaining functional properties, ${ }^{4,5,6,7,8,9,10}$ however, many questions remain regarding the intricate interplay between structural distortions and the ligation of small adducts. Here we report a molecular-level investigation on the interaction of carbon monoxide with simple Fe and Co tetraphenyl-porphyrin model systems adopting a distinct saddle-shape conformation, one of the prominent nonplanar macrocycle geometries, following adsorption on a smooth metal surface. ${ }^{11,12}$ Our scanning tunneling microscopy and molecular manipulation experiments reveal a surprising carbonyl binding scheme that contrasts the common wisdom of exclusive axial monocarbonyl ligation at biological or related synthetic porphyrin species. Density functional theory calculations for both adsorbed and isolated Co-tetraphenyl-porphyrin demonstrate that $\mathrm{CO}$ in fact can bridge specific pyrrole groups with the metal center, which effect is induced by the saddle-shape conformation and explains the observed mono- and cis-dicarbonyl geometries. With our findings a novel ligation scheme for $\mathrm{CO}$ at metalloporphyrins is established that deserves to be considered in general regarding its potential influence on the groups' functionality.

Despite the multitude of studies focusing on the binding mechanism of diatomic molecules at the heme prosthetic group, ${ }^{13}$ and the related relevance of $\mathrm{CO}$ as cellular signaling molecule, ${ }^{14,15}$ the factors regulating the affinity and geometry of $\mathrm{CO}$ remain to be fully elucidated. In the context of the discrimination between $\mathrm{CO}$ of endogenous origin and dioxygen many explanations invoke the local environment of the prosthetic group acting specifically on the ligated molecules via steric, hydrogen bonding or electrostatic interactions. ${ }^{1,16,17,18}$ For the analysis it is generally assumed that monocarbonyl axial ligation takes place right at the metal center, ${ }^{1}$ whereby distortions of the functional group may occur in the presence of the adduct. This calls for investigations probing directly the interaction of $\mathrm{CO}$ with model porphyrin systems, where the conformation of the macrocycle is controlled and a surrounding is provided 
that precludes the interference of additional protein or other units. We realized such an elementary portrayal by confining the species Co- and Fe-tetraphenyl-porphyrin (CoTPP, FeTPP) on well-defined $\mathrm{Cu}(111)$ and $\mathrm{Ag}(111)$ surfaces. By the surface anchoring arrays of coordinatively unsaturated species are generated with a known macrocycle conformation, ${ }^{11,12}$ whence their response upon exposure to $\mathrm{CO}$ can be investigated in situ using scanning tunneling (STM) microscopy.

\section{Identification of $\mathrm{CO}$ ligation mode at adsorbed CoTPP and FeTPP}

An exemplary STM image of a single CoTPP molecule is reproduced in fig. 1a, along with a model highlighting the distortion of the macrocycle, which significantly differs from the structure of the molecule in $3 \mathrm{D}$ crystallites showing only minor ruffling distortions of the porphinato skeleton. ${ }^{19}$ The saddle-shape conformation of the CoTPP macrocycle is similar on both $\operatorname{Ag}(111)$ and $\mathrm{Cu}(111)$ substrates with pairs of opposite

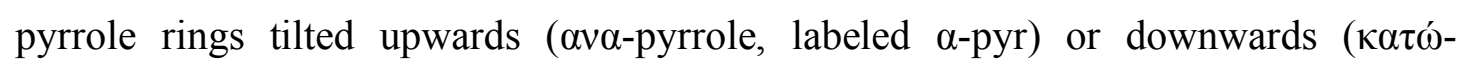
pyrrole, labeled $\kappa$-pyr) by an angle $\rho$ of $20-30^{\circ} .{ }^{11,12}$ Moreover, the phenyl substituents are rotated by the dihedral angle $\left(\theta \approx 45^{\circ}\right)$ out of the surface plane. The bending of opposing pyrrole groups leads to a characteristic imaging of the macrocycle as a triple protrusion when occupied states are probed. ${ }^{11,12,20}$ Its core part reflects the metal center, which is flanked by the two $\alpha$-pyr moieties. 

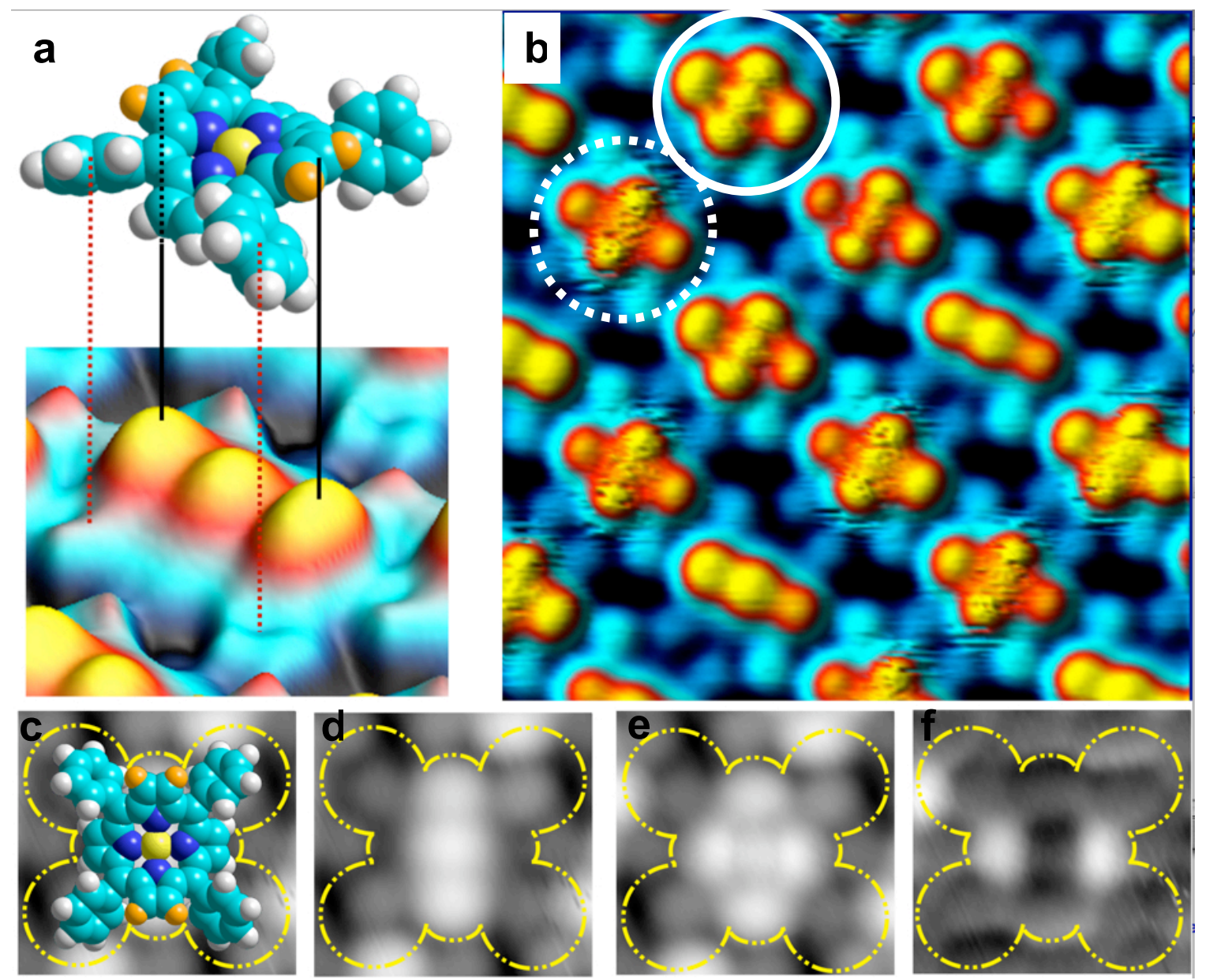

Figure 1 Saddle-shape conformation of CoTPP on $\mathrm{Ag}(111)$ response towards CO exposure. a Conformational adaptation of CoTPP adsorbed on the (111) surfaces of copper or silver. The pseudo 3D rendering of a high-resolution STM image shows the saddle-shaped macrocycle distortion and alternately rotated phenyl groups $(V=-0.7 \mathrm{~V}, I=0.46 \mathrm{nA})$. Pyrrole hydrogens pointing away from the surface ( $\alpha$-pyr moiety) are emphasized in orange colour. $\mathbf{b}$ CoTPP array on $\mathrm{Ag}(111)$ following exposure to $11 \mathrm{~L}$ CO. There is a mixed layer comprising CoTPP, CO/CoTPP (dashed circle) and $(\mathrm{CO})_{2} /$ CoTPP (solid circle). c Top-view model of CoTPP overlaid on STM image. d Top-view topography of undecorated CoTPP and (e) dicarbonyl species. $f$ The corresponding difference image emphasizes the $\approx 5.3 \AA$ between co-related maxima.

On $\operatorname{Ag}(111)$, CoTPP forms regular arrays (see Fig. 1b). For the carbonyl attachment we exposed the sample to $\mathrm{CO}$ background pressures in situ. Following the dosage of small amounts of $\mathrm{CO}$ the array structure remains unaffected and the intramolecular features are still clearly resolved (this holds for the entire low-coverage regime investigated, as shown in the data series included in the Supplementary Information). However, a fraction of the CoTPP is decorated by two additional protrusions, located at both sides of the Co center, respectively, at the $\kappa$-pyr groups. Thus selective uptake of $\mathrm{CO}$ adducts occurred, resulting in a cross-like appearance of the porphyrin macrocycle. A closer inspection of Fig. 1b reveals that two similar CO-induced 
configurations are present: The first species (solid circle) shows five distinct protrusions and represents a stable entity, which is called a static cross. The second structure is topographically similar, but the CO-related features are imaged scraggy (cf. dashed circle in fig. 1b), which is an indication of rapid fluctuations with a frequency exceeding that employed for the measurement. This is called a labile cross.
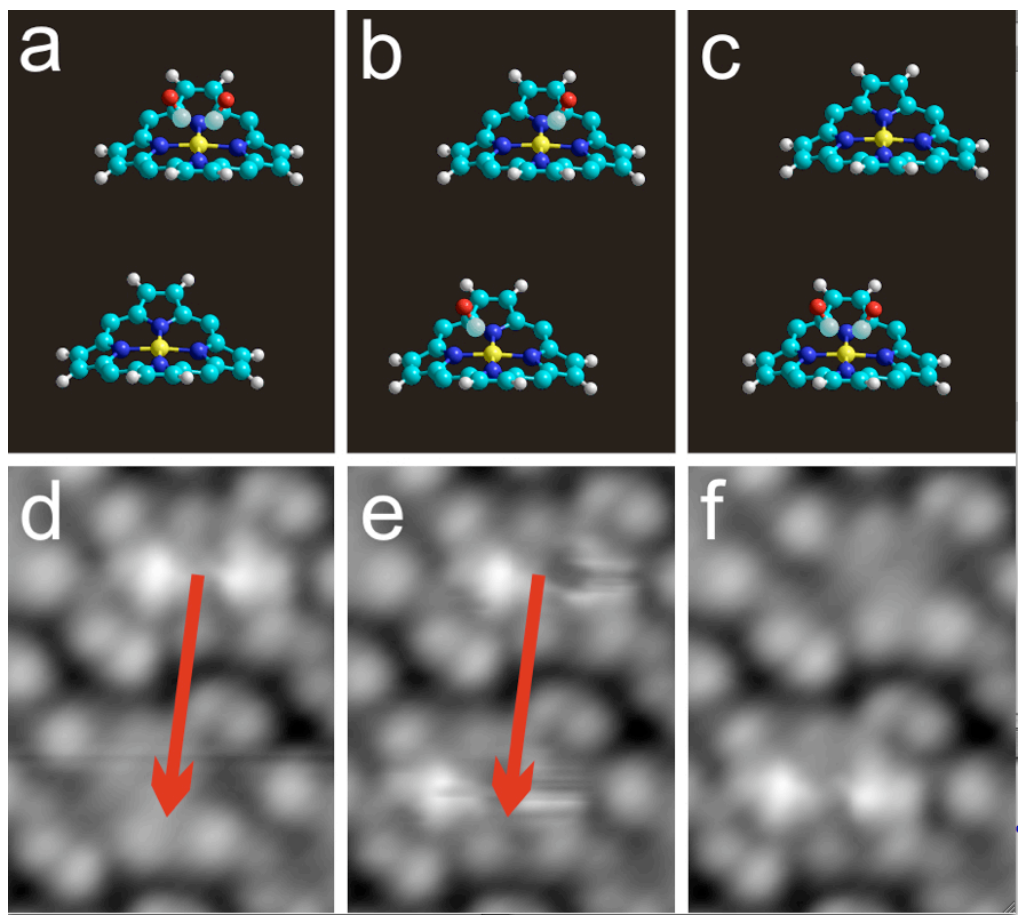

Figure 2 Transfer of carbonyl ligands by molecular manipulation. a-c Schematic model of two saddle-shaped porphyrin macrocycles with different ligation modes of two $\mathrm{CO}$ molecules (meso-phenyl groups are omitted for clarity) corresponding to the data sequence below. d-f STM images of CoTPP at $500 \mathrm{mV}$ sample bias demonstrating the sequential transfer of two $\mathrm{CO}$ adducts. The lateral manipulation steps are marked by red arrows indicate the route of the STM tip converting a pristine CoTPP firstly to CO/CoTPP and subsequently $(\mathrm{CO})_{2}$ /Co-TPP.

We unambiguously clarified the nature of the two distinct cross-shaped species by molecular manipulation experiments, using the STM tip as a tool to translate adsorbates across a surface, as successfully demonstrated for $\mathrm{CO}$ molecules at metal substrates. ${ }^{21,22,23}$ Firstly, we positioned the tip above a static cross (stabilized at 500 $\mathrm{mV}$ and $30 \mathrm{pA}$, see Fig. 2a/d) and lowered the tip towards the surface by increasing the current to $1 \mathrm{nA}$. Subsequently, the tip was laterally moved to an adjacent CoTPP molecule and finally retracted to its initial height. The following STM image shows that this procedure converts one static into two labile crosses (Fig. 2b/e). Applying the same procedure again, it is possible to reunite the two unstable species to one static 
cross, now localized at the neighboring molecule (Fig. 2c/f). Single CO-related features can also be abstracted and irreversibly desorbed by applying higher voltage pulses. Hence the static configuration must be composed of two $\mathrm{CO}$ molecules attached to a single CoTPP, and represents a cis-dicarbonyl species. Accordingly the labile cross is a related monocarbonyl complex. The protrusions associated with the $\mathrm{CO}$ adducts (cf. figs. 1c-f). occupy distinct, symmetric positions at the macrocycle $\kappa$ pyr moieties, which effect must be related to the porphyrin conformation. When there is only one $\mathrm{CO}$ attached, the carbonyl ligand is rapidly flipping between two equivalent sites, indicating that the corresponding energy barrier is rather low. Consequently, effects of the proximity of the STM tip on the flipping cannot be excluded. The slight asymmetry of the flipping carbonyl ligands imaging in fig. 2e is hence ascribed to tip-sample interactions; however, it was not possible to maintain it at a specific location in the manipulation experiments.

The unusual carbonyl ligation is not limited to the system CoTPP/Ag(111). It similarly appears with iron centers, as demonstrated by the STM image in Fig. 3a. For this experiment, we synthesized FeTPP directly on the $\operatorname{Ag}(111)$ surface by direct metalation of the macrocycle. ${ }^{20}$ The generated FeTPP array was exposed to CO following the same experimental protocols as in the CoTPP case. The additional insitu dosage of $\mathrm{CO}$ leads again to features with characteristics similar to those described above (cf. Fig. 3a). Both static and labile crosses, corresponding to $\mathrm{CO} / \mathrm{FeTPP}$ and $(\mathrm{CO})_{2} / \mathrm{FeTPP}$, are observed. Furthermore, the same procedures were conducted with a different underlying metal surface: $\mathrm{Cu}(111)$, showing a smaller lattice parameter and higher chemical reactivity. Nonetheless, the characteristic MTPP-carbonyl features reappear, as demonstrated with the STM image in Fig. 3b for the specific configuration of $(\mathrm{CO})_{2} / \mathrm{CoTPP} / \mathrm{Cu}(111)$. In all cases manipulation experiments could be successfully conducted to distinguish the respective carbonyl species.

These experimental findings let us conclude that the asymmetric monocarbonyl and symmetric cis-dicarbonyl attachment is potentially a universal feature for the saddleshaped metallo-porphyrin conformers. Whereas dicarbonyl ligation in cis geometry has been invoked for early transition metal porphyrins, notably with Mo, it is definitely unexpected for the case of Fe or Co centers. ${ }^{24,25}$ The reason is the difference in electronic structure and the atomic size that results in sizable displacements for Mo 
centers out of the porphyrin plane, eager to bind adducts in a multiple ligation mode. The present saddle-shape conformation could support a related ligation scheme, however, when we compare the STM topography of the observed species with dicarbonyl species generated at single adsorbed Fe or Co atoms major differences appear; notably the distance between CO-related features at the present MTPPs significantly exceeds that for the adatom dicarbonyls. ${ }^{22,26}$

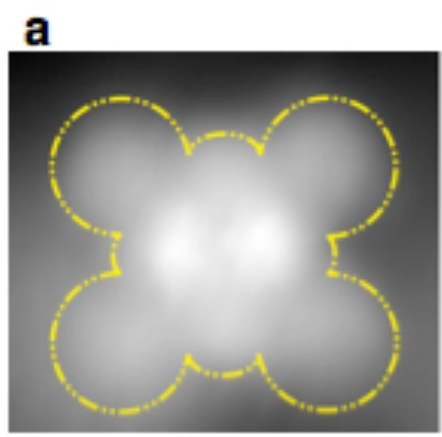

b
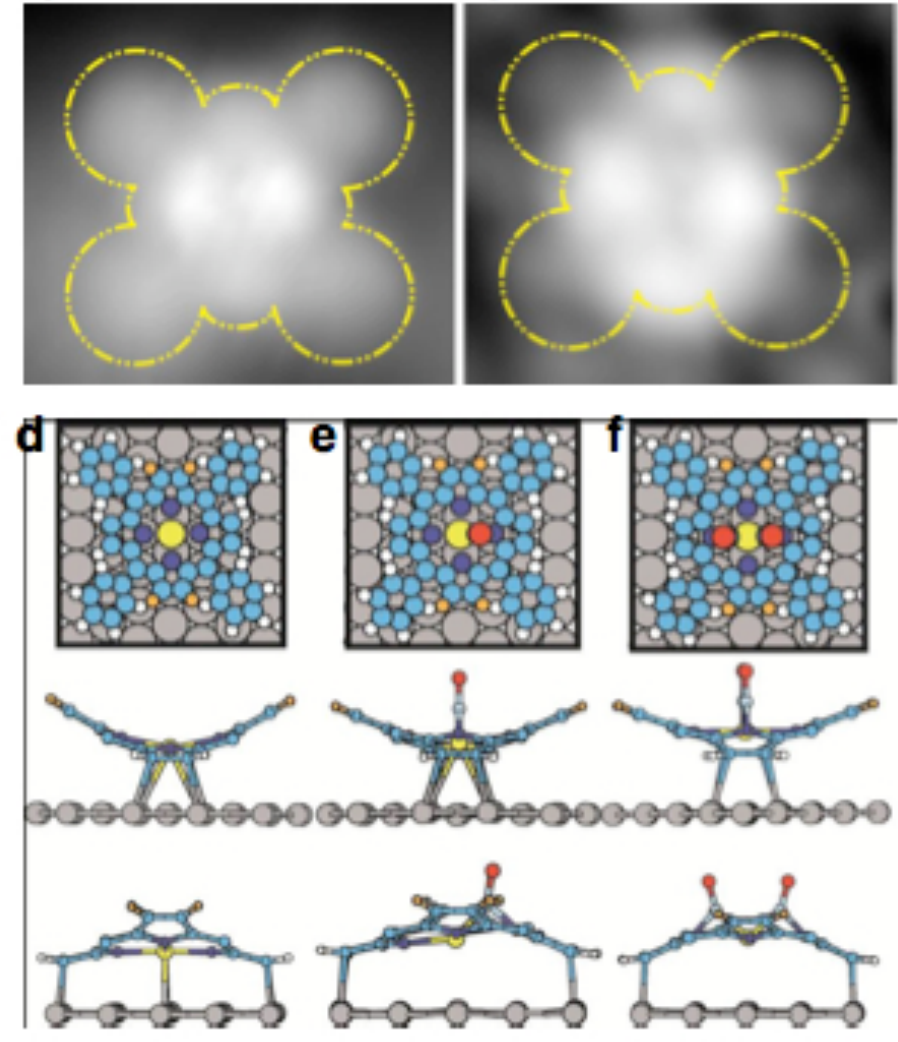

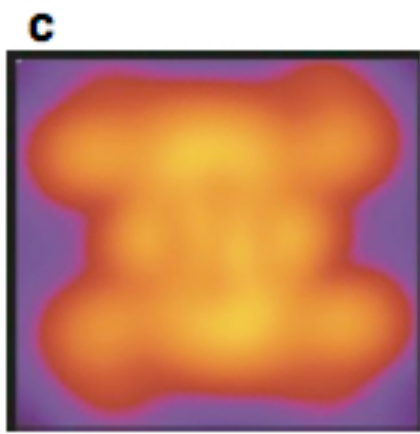

g
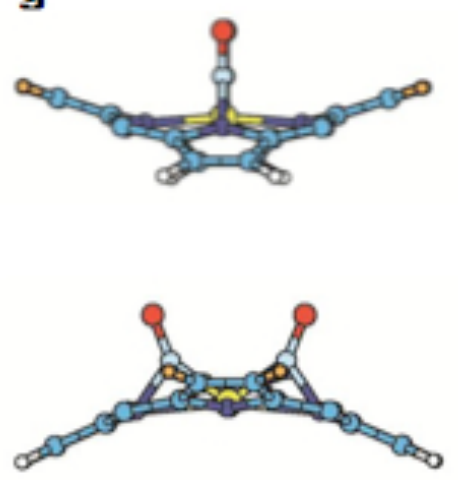

Figure 3 Comparison of experimental data and theoretical description. STM images of a $(\mathrm{CO})_{2} / \mathrm{Fe}-\mathrm{TPP}$ on $\mathrm{Ag}(111)$ and $\mathbf{b}(\mathrm{CO})_{2} / \mathrm{Co}-\mathrm{TPP}$ on $\mathrm{Cu}(111)$ c The image simulation for $(\mathrm{CO})_{2}$ /Co-TPP with two $\mu$-carbonyl adducts on $\mathrm{Cu}(111)$ from DFT reproduces the main features of the experimental STM data. d-f DFT modeling showing the relaxed geometry of the systems Co-TPP (d), CO/Co-TPP (e) and (CO) $)_{2} / \mathrm{Co}$-TPP (f) on $\mathrm{Cu}(111)$ with bridging $\mu \mathrm{CO}$ ligation. g DFT model of stable, free $(\mathrm{CO})_{2} / \mathrm{Co}$-TPP species in cis- $\mu$-dicarbonyl geometry.

\section{Theoretical analysis of $\mathrm{CO}$ adducts at saddle-shaped CoTPP conformers}

In order to rationalize the interplay between macrocycle conformation and $\mathrm{CO}$ ligation, we performed extensive density functional theory (DFT) calculations. Regarding the situation with surface-confined MTPP-carbonyls, we concentrate on 
the CoTPP/Cu(111) system. Prior to adding the ligand, the CoTPP is placed in the experimentally determined bridge position on $\mathrm{Cu}(111)$. The relaxed molecule displays significant bonding with the metal substrate. Interestingly, the connecting (or chemically bonded) areas are strongly localized due the non-planarity of the porphyrin molecule. There are three distinct locations: a strong $\mathrm{Co}-\mathrm{Cu}$ bond (distance of $2.8 \AA$ ) and the di- $\sigma$ bonding of two $\mathrm{C}=\mathrm{C}$ bonds closest to the surface from the $\kappa$ pyr groups (C-Cu length $2.2 \AA$ ). In the adsorbed state, cobalt porphyrin retains a saddle-shape conformation. We considered several binding geometries for the carbonyl adducts, and the results definitely exclude a cis-dicarbonyl ligation at the metal center. However, we found that the bending of the macrocycle yields a different option: a carbonyl ligand $(\mu \mathrm{CO})$ that bridges the coordination bond between the iminic $(-\mathrm{C}=\mathrm{N}-)$ nitrogen and metal center. This ligand insertion scheme is favored exclusively at the $\kappa$-pyr groups, both for steric reasons and because of the weaker dative $\mathrm{Co}-\mathrm{N}_{\kappa}$ bonds. Thus, the bridging position permits to bind two $\mathrm{CO}$ adducts at two opposed and equivalent sites of the porphyrin molecule. The corresponding STM image simulation for the dicarbonyl species (cf. Fig. 3c) reproduces the main characteristics of the experimental topography, nicely rendering the carbonyl-related features.

With the $\mu$-carbonyl ligand bonded to the macrocycle, there is a modification of the Co-porphyrin adsorption structure in two concomitant ways as seen in Fig. 3d-f: the vertical lifting of the central Co atom and the rupture of the coupling with the metal substrate. However one readily notices that the saddle-shape geometry is overall conserved. For one CO bridging ligand the effect is subtle and localized to the central cation: Co undergoes a small vertical shift toward CO, weakening its bonding to the substrate but the $\kappa-p y r$ subunits remain anchored to the substrate. Here, the calculations (cf. Fig. 3, phenyl substituents not shown) reveal a sizeable stretching of the Co-N distance to $2.2 \AA$ underneath the carbonyl ligand. Upon attachment of two carbonyls, a symmetrical adsorption structure is achieved: now the central cation is detached from the surface (the Co-Cu distance increases to $3.5 \AA$ ) whereas the $\kappa$-pyr groups remain linked to the surface via a pronounced tilt of the pyrrole plane. A somewhat related behaviour occurs with NO ligation to CoTPP adsorbed on $\operatorname{Ag}(111)$, where the system response includes also a suppression of the Co-substrate interaction. ${ }^{27}$ With the present system this strongly signals that the underlying surface 
is not directly determining the $\mathrm{CO}$ ligation mode, i.e., it is not a result of a surface coordination architecture. $^{28}$

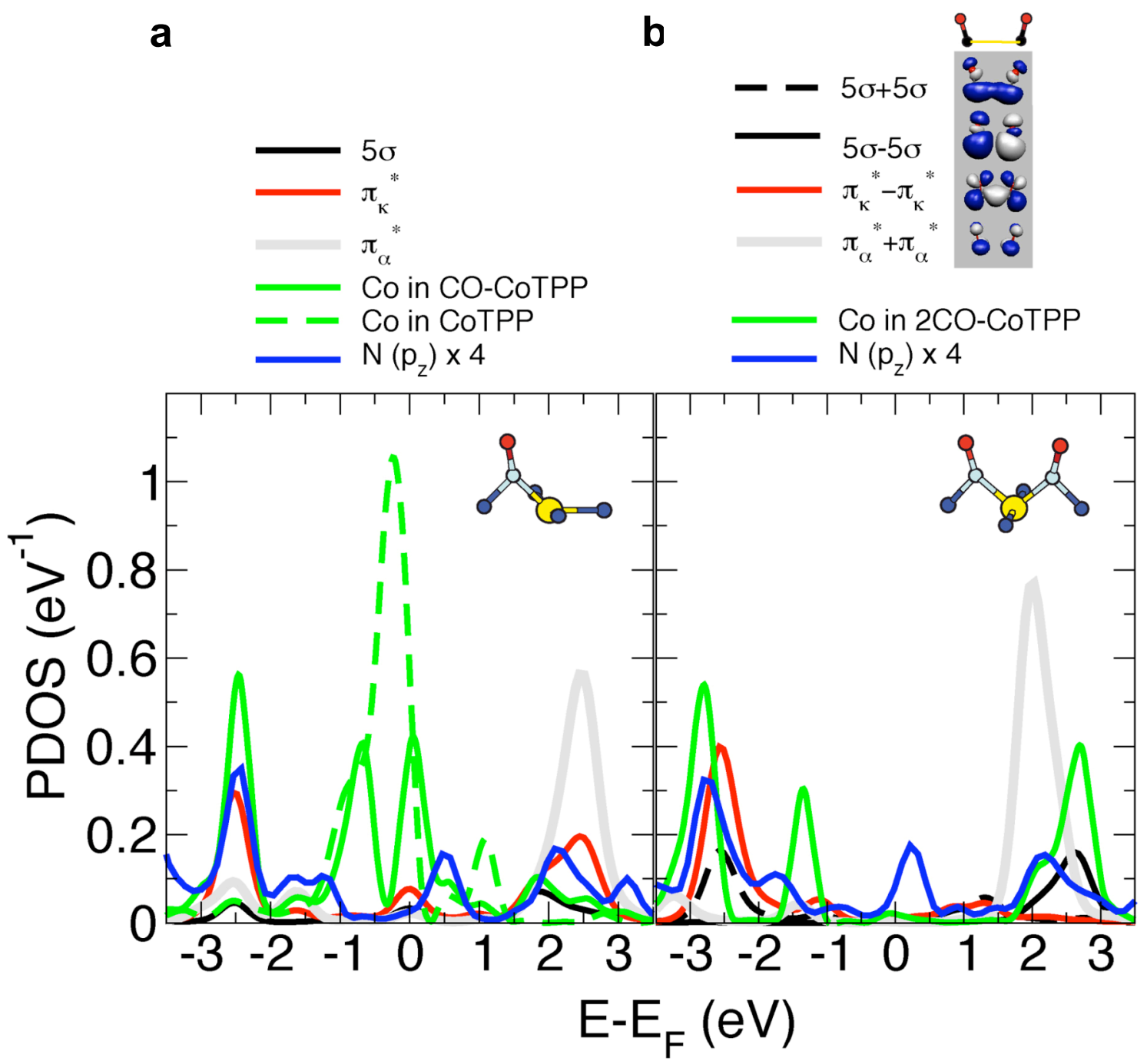

Figure 4 Electronic properties of the bridged carbonyl complexes. Projected density of states (PDOS) of a CO/Co-TPP and $\mathbf{b}(\mathrm{CO})_{2} / \mathrm{Co}$-TPP adsorbed on $\mathrm{Cu}(111)$ onto the molecular states of the $\mathrm{CO}$ adducts and the active atomic states of $\mathrm{Co}$ and $\mathrm{N}$ atoms $\left(\mathrm{d}_{\mathrm{xz}}\right.$ Co and $p_{z} N$ with $z$ the normal direction to the surface and $x$ aligning with $C_{0}-N_{\kappa}$ bond). The degenerate $2 \pi^{*}$ states along the $N_{\alpha}$ and $N_{\kappa}$ pyrrole subunits are indicated. The dimer molecular states are sketched and labeled with the combination of monomer states.

In agreement, the novel complexation of two $\mathrm{CO}$ entities pertains similarly to gas phase environment as predicted by the DFT structure of the free complex (cf. Fig. $3 \mathrm{~g}$ ). Subtle changes can be noticed, however, on the $\mathrm{C}$ atoms of the $\kappa$-pyr groups switching from sp3 hybridization for the adsorbed to $\mathrm{sp} 2$ for the isolated species. Note that for isolated species in saddle-shape geometry furthermore the bonding of carbonyl moieties on both opposite sides of the macrocycle plane cannot be excluded. 
The formation of the carbonyl bridging complex is possible thanks to the chemical interaction between the two carbonyl ligands mediated by the Co center. The two carbonyl ligands can be actually considered as a dimer entity linked through the cobalt center as sketched in the orbital diagram in Fig. 4. The plot shows the calculated density of states projected onto the molecular states of the ligands for one (Fig. 4a) and two bridging carbonyls (Fig. 4b), respectively. The $\mathrm{CO}$ frontier orbitals are the $5 \sigma(\mathrm{HOMO})$ and the degenerate $2 \pi^{*}$ states (LUMO). On the bridging site on the molecule, the $2 \pi^{*}$ states split and we mark them as $2 \pi_{\alpha}{ }^{*}$ and $2 \pi_{\kappa} *$ if they point to

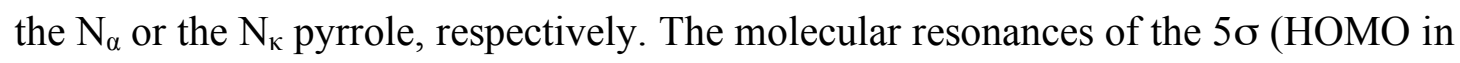
solid dark Fig. 4a) and $2 \pi_{\kappa} *$ (LUMO in solid red Fig. 4a) states are further shifted and broadened in energy due to their strong orbital overlap with the corresponding pair of $\mathrm{Co}$ and $\mathrm{N}$ atoms of the adsorbed porphyrin molecule. Such energy shifting and broadening imply that the $5 \sigma$ level becomes partially empty and the $2 \pi_{\kappa}{ }^{*}$ partially occupied, corresponding to the donation-back donation bonding mechanism. For two $\mathrm{CO}$ ligands, the molecular states sketched in Fig $4 \mathrm{~b}$ (right) resemble that of a molecular dimer exhibiting a reduced HOMO-LUMO gap as compared to the monomer case and thus reinforcing the bonding to the cobalt porphyrin. The antibonding combination of the $5 \sigma$ (solid black) and the bonding combination of the $2 \pi_{\kappa} *$ (solid red), acting as HOMO and LUMO states of the $(\mathrm{CO})_{2}$ dimer, remain localized states but are respectively up- and down- shifted relative to the Fermi level, as a result of a complete donation and backdonation stabilizing the dimer on the porphyrin molecule.

Figure 4 furthermore contains the projected DOS (PDOS) onto characteristic $d$ Co and $p \mathrm{~N}$ atomic orbitals. Without ligation by CO molecules by CoTPP, the Co $d_{\mathrm{xz}}$ PDOS is largely occupied (green dashed line). As one CO molecule is added, the $d_{\mathrm{xz}}$ orbital (green line in left panel of Fig.4) mainly rehybridizes with the $5 \sigma$ and $2 \pi$ of the bonded molecule giving rise to common peaks coincidental in energy at -2.5 and $+2.5 \mathrm{eV}$ with respect to the Fermi level. These peaks agree well with the classical image of CO charge donation and back-donation (cf. Supplementary Information for further details; note that the $d_{\mathrm{yz}}$ orbital is much less affected as expected from the binding geometry). The same is true for the peaks in the PDOS of the $p_{z}$ orbital of the closest nitrogen atom. A second CO molecule leads to stronger rehybridization of the Co electronic structure marked by the enhanced unoccupied $d$ peak at $+2.5 \mathrm{eV}$, while 
keeping the N-based electronic structure basically unchanged with respect to the mono-carbonyl case.

The calculated PDOS onto the Co $d$ manifold completed by a Mulliken-like charge analysis provide relevant information on the charge state of the adsorbed Co atom before and after successive CO ligation (see Supplementary Information). It can be inferred that the $\mathrm{Co}$ (II) state of free CoTPP reduces to a $\mathrm{Co}(\mathrm{I})$ state upon adsorption on $\mathrm{Cu}(111)$ due to the coupling between $\mathrm{Co} d$-states and the $\mathrm{Cu}$ substrate. The adsorbed molecule is hence in a nonmagnetic singlet spin state.

The monocarbonyl ligation does not change the oxidation state of adsorbed Co, albeit the electronic structure is modified rendering a doublet spin state. In contrast the cisdicarbonyl ligation again quenches the spin as a result of larger charge donation. The electronic characteristics of the adsorbed $\operatorname{CoTPP}(\mathrm{CO})_{2}$ species are consistent with a $\operatorname{Co}(0)$ oxidation state.

We also performed a set of preliminary model calculations for the case of Fe centers, indicating differences compared to the results described above, but also signaling the possibility of bridged ligation. Note that the case of iron porphyrins is more diffcult to handle because of the higher complexity regarding modeling and experimental assessment of the Fe center. Furthermore a comparative assessment with the ligation of NO revealed that here the expected conventional axial monocoordination pathway prevails, which is in perfect agreement with a recent study using space-averaging methods. ${ }^{27}$ These results will be presented in full detail elsewhere.

\section{Conclusion}

We identified a unique ligation mode for $\mathrm{CO}$ adducts at surface-supported cobalt and iron porphyrins by combined molecular-level STM observations and theoretical modeling. The saddle-shape conformation of both Fe and Co-tetraphenylporphyrins induces notably a cis-carbonyl geometry, with $\mathrm{CO}$ binding to two spatially distinguished sites. Detailed calculations with Co centers reveal that CO bridges specific pyrrole groups with the metal atom either as asymmetric monocarbonyl or symmetric cis-dicarbonyl species. This bonding scheme for $\mathrm{CO}$ adducts may be of general relevance regarding the chemical nature and functional behavior of porphyrins with late transition metal centers in situations where conformational distortions interfere. 
Acknowledgements Work supported by the ERC Advanced Grant MolArt $\left({ }^{\circ} 247299\right)$, the DFG Cluster of Excellence Munich Center for Advanced Photonics, Canadian NSERC and CFI. A.W.-B. and J.R. thank the German Academic Exchange Service and the Deutsche Forschungsgesellschaft for scholarships, respectively. MLB acknowledges the computational time at the Leibnitz Rechenzentrum Garching. NL thanks Spanish MICINN for financial support, grant FIS2009-1271-C04-01.

Author contributions K.S., W.A., A. W.-B. \& J.R. performed the STM experiments, analyzed and interpreted the experimental data. The theoretical analysis was provided by M.-L. B. and N. L.. J.V.B., W.A. \& M.-L. B. conceived the studies and co-wrote the paper with K.S. \& N.L..

\section{Methods}

The experiments were performed using a custom-designed STM housing a commercial LTSTM (for details see www.lt-stm.com and ref. ${ }^{23}$ ). The system base pressure was below $2 \mathrm{x}$ $10^{-10}$ mbar and all measurements were performed at $6 \mathrm{~K}$ to obtain high stability and highresolution topographic images. The employed $\mathrm{Ag}(111)$ and $\mathrm{Cu}(111)$ substrate were prepared by standard procedures (cycles of $\mathrm{Ar}^{+}$sputtering and annealing) to obtain extended flat terraces separated by monatomic steps. The etched tungsten tip is prepared by Argon bombardment and silver or copper coated by controlled dipping into the bare surface. All STM images were taken in constant current mode and treated by the WsXM program (www.nanotec.es). Molecules were deposited by organic molecular beam epitaxy (OMBE) from a quartz crucible held at $625 \mathrm{~K}$ for Co-TPP and $575 \mathrm{~K}$ for $2 \mathrm{H}-\mathrm{TPP}$ with a typical rate below one monolayer per hour and at a background pressure in the range of $10^{-10} \mathrm{mbar}$. FeTPP was synthesized in situ by exposing a H2-TPP submonolayer to an atomic beam of Fe (purity $99.998 \%$ ). Because the additional attachment of CO (purity $99.97 \%$ ) was not possible at elevated temperatures, the sample was exposed to $\mathrm{CO}$ doses directly in the measurement position at $\mathrm{T}=6 \mathrm{~K}$, increasing up to $\sim 20 \mathrm{~K}$ during the dosage.

DFT: The calculations have been performed using the projected augmented wave (PAW) method within the local density approximation (LDA) as implemented in the VASP code. ${ }^{29}$ We employ a $400 \mathrm{eV}$ energy cutoff and spin polarization. The periodic slab calculations are $\Gamma$-point only for a $11 \times 5 \operatorname{sqrt}(3)$ unit cell, accounting for the diluted phase of porphyrins (approximately the single-molecule limit). The $\mathrm{Cu}$ slab is modeled by three atomic monolayers and the vacuum space between adjacent slabs corresponds to eight atomic monolayers. The surface $\mathrm{Cu}$ layer and all adsorbate atoms were relaxed until atomic forces were smaller than $0.05 \mathrm{eV} / \AA$.

The molecule-surface interaction for this large $\pi$-orbital system is probably driven by van der Waals interactions, especially in the ligated case when the chemical contact between the metallic center and the metallic substrate is disrupted. Popular exchange-correlation functionals suffer from their deficiency of non-local correlation terms including the dispersion energy. We use here LDA as a partially satisfactory solution for large-scale adsorption systems, on which further improvement like adding the dispersive interactions in a 
self-consistent way is not feasible yet. ${ }^{30}$ Calculations in LDA have yielded good results regarding the adsorption of large $\pi$-orbital molecular systems, thanks to an approximate compensation for the lack of van der Waals interactions by the LDA tendency to overestimate binding energies. ${ }^{12,31,32,33,34}$

\section{References}

1 Spiro, T. G. \& Kozlowski, P. M. Is the CO Adduct of Myoglobin Bent, and Does It Matter? Acc. Chem. Res. 34, 137-144 (2001).

2 Collman, J. P., Boulatov, R., Sunderland, C. J. \& L.Fu. Functional Analogues of Cytochrome c Oxidase, Myoglobin, and Hemoglobin. Chem. Rev. 104, 561588 (2004).

Ghosh, A. Metalloporphyrin-NO bonding: Building bridges with organometallic chemistry. Acc. Chem. Res. 38, 943 - 954 (2005).

Hoard, J. L. Stereochemistry of Hemes and Other Metalloporphyrins. Science 174, 1295-1302 (1971).

Kratky, C. et al. The Saddle Conformation of Hydroporphinoid Nickel(II) Complexes: Structure, Origin, and Stereochemical Consequences. Helv. Chim. Act. 68, 1312-1327 (1985).

Barkigia, K. M., Chantranupong, L., Smith, K. M. \& Fajer, J. Structural and Theoretical Models of Photosynthetic Chromophores. Implications for Redox, Light Absorption Properties and Vectorial Electron Flow. J. Am. Chem. Soc. 110, 7566-7567 (1988).

Barkigia, K. M. et al. Nonplanar Porphyrins. X-ray Structures of $(2,3,7,8,12,13,17,18$-Octaethyl- and -Octamethyl-5,10,15,20tetraphenylporphinato)zinc(II). J. Am. Chem. Soc. 112, 8851-8857 (1990). Sparks, L. D. et al. Metal dependence of the nonplanar distortion of octaalkyltetraphenylporphyrins. J. Am. Chem. Soc. 115, 581-592 (1993). Shelnutt, J. A. et al. Nonplanar porphyrins and their significance in proteins. Chem. Soc. Rev. 27, 31-41 (1998). Senge, M. O. Exercises in molecular gymnastics - bending, stretching and twisting. Chem. Comm., 243 - 256 (2006). Weber-Bargioni, A. et al. Visualizing the frontier orbitals of a conformationally adapted metalloporphyrin. ChemPhysChem 9, 89-94 (2008). Auwärter, W. et al. Site-specific electronic and geometric interface structure of Co-tetraphenyl-porphyrin layers on Ag(111). Phys. Rev. B 81, 245403 (2010).

Springer, B. A. \& Stephen G. Sligar, J. S. O., George N. Jr. Phillips. Mechanisms of Ligand Recognition in Myoglobin. Chem. Rev. 94, 699 - 714 (1994).

Aono, S. Biochemical and Biophysical Properties of the CO-Sensing Transcriptional Activator CooA. Acc. Chem. Res. 36, 825-831 (2003).

Kim, H. P., Ryter, S. W. \& Choi, A. M. K. CO as a cellular signaling molecule. Annu. Rev. Pharmacol. Toxicol. 46, $411-449$ (2006). Inhibition of CO Binding to Heme Proteins. Science 284, 473 - 476 (1999).

Sigfridsson, E. \& Ryde, U. On the significance of hydrogen bonds for the discrimination between $\mathrm{CO}$ and $\mathrm{O}_{2}$ by myoglobin. J. Biol. Inorg. Chem. 4, 99 110 (1999). 
Leu, B. M. et al. Quantitative Vibrational Dynamics of Iron in Carbonyl Porphyrins. Biophys. J. 92 3764-3783 (2007).

Madura, P. \& Scheidt, W. R. Stereochemistry of Low-Spin Cobalt Porphyrins. 8. $\alpha, \beta, \gamma, \delta$-Tetraphenylporphinatocoba(II). Inorg. Chem. 15, 3182-3184 (1976).

Auwärter, W. et al. Controlled metalation of self-assembled porphyrin nanoarrays in two dimensions. ChemPhysChem 8, 250-254 (2007).

Meyer, G., Neu, B. \& Rieder, K. H. Controlled lateral manipulaiton of single molecules with the scanning tunneling microscope. Appl. Phys. A 60, 343-345 (1995).

Lee, H. J. \& Ho, W. Single-bond formation and characterization with a scanning tunneling microscope. Science 286, 1719-1722 (1999).

Auwärter, W. et al. Molecular nanoscience and engineering on surfaces. Int. J. Nanotechnol. 5, 1171-1193 (2008).

Brand, H. \& Arnold, J. Recent developments in the chemistry of early transition metal porphyrin compounds. Coord. Chem. Rev. 140, 137 - 168 (1995).

Smith, P. D., James, B. R. \& Dolphin, D. H. Structural aspects and coordination chemistry of metal porphyrin complexes with emphasis on axial ligand binding to carbon donors and mono- and diatomic nitrogen and oxygen donors. Coord. Chem. Rev. 39, 31 - 75 (1981).

Wahl, P. et al. Kondo Effect of Molecular Complexes at Surfaces: Ligand Control of the Local Spin Coupling. Phys. Rev. Lett. 95, 166601 (2005).

Flechtner, K., Kretschmann, A., Steinrück, H. P. \& Gottfried, J. M. NOinduced reversible switching of the electronic interaction between a porphyrincoordinated cobalt ion and a silver surface. J. Am. Chem. Soc. 129, 12110 12111 (2007).

Barth, J. V. Fresh perspectives for surface coordination chemistry. Surf. Sci. 603, 1533-1541 (2009).

Kresse, G. \& Joubert, D. From ultrasoft pseudopotentials to the projector augmented-wave method. Phys. Rev. B 59, 1758 - 1775 (1999).

Mercurio, G. et al. Structure and Energetics of Azobenzene on $\operatorname{Ag}(111)$ : Benchmarking Semiempirical Dispersion Correction Approaches. Phys. Rev. Lett. 104, 036102 (2010).

Vladimirova, M. et al. Substrate-induced supramolecular ordering of functional molecules : theoretical modelling and STM investigation of the PEBA/Ag(111) system. Acta Mater. 52, 1589-1596 (2004).

Rohlfing, M., Temirov, R. \& Tautz, F. S. Adsorption structure and scanning tunneling data of a prototype organic-inorganic interface: PTCDA on Ag(111). Phys. Rev. B 76, 115421 (2007).

Klappenberger, F. et al. Conformational adaptation in supramolecular assembly on surfaces. ChemPhysChem 8, 1782-1786 (2007).

Franke, K. J. et al. Reducing the Molecule-Substrate Coupling in $\mathrm{C}_{60}$-Based Nanostructures by Molecular Interactions. Phys. Rev. Lett. 100, 036807 (2008). 


\section{Table of Content Graphic (TOC)}

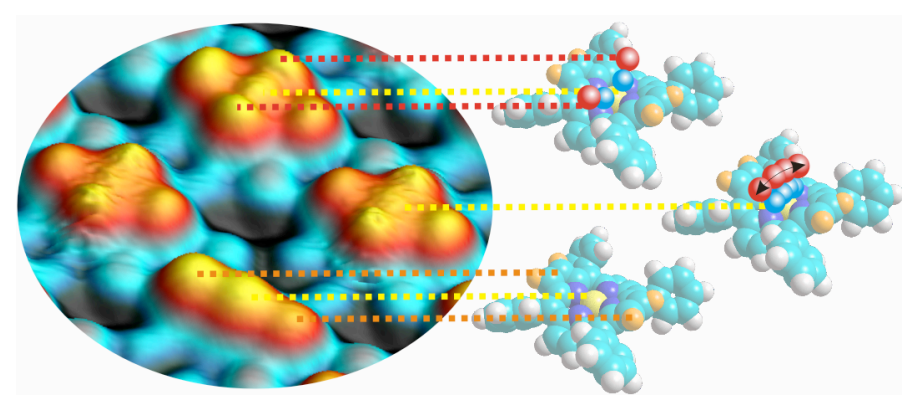

Novel bonding mechanism of carbonyl ligands at surface confined $\mathrm{Fe}$ - and Coporphyrins identified by direct tunneling microscopy observations and rationalized by density functional theory modeling. Carbonyl adducts at saddle-shaped conformers bridge downward bent pyrrole groups with the metal atom either as asymmetric monocarbonyl or symmetric cis-dicarbonyl species. 